\title{
Mild encephalopathy with reversible extensive white matter lesions in a child with acute adenoviral infection and a literature review
}

\author{
Mustafa Emre Akın ${ }^{1 \oplus}$, Ayşegül Neşe Çıtak Kurt² ${ }^{2 \odot}$ Gülsüm İclal Bayhan ${ }^{3 \odot}$, Zeynep \\ Dinçer Ezgü̈${ }^{4 \oplus}$, Sevtap Şimşek Bulut ${ }^{1 \oplus}$ \\ ${ }^{1}$ Department of Radiology and Divisions of ${ }^{2}$ Pediatric Neurology and ${ }^{3}$ Pediatric Infection, ${ }^{4}$ Department of Pediatrics, Ankara, Yildirım \\ Beyazıt University Faculty of Medicine, Yenimahalle Training and Research Hospital, Ankara, Turkey.
}

\begin{abstract}
Background. Mild encephalopathy with a reversible splenial lesion (MERS) is a known clinical-radiological description. However, MERS with extensive lesions (MERS type-2) is rarely associated with adenovirus. There are only three published cases of MERS type-2 associated with adenovirus infection.

Case. We present a 10-year-old previously healthy girl who presented with speech difficulty and mild encephalopathy after three days of prodromal illness. The magnetic resonance imaging (MRI) revealed bilateral diffusion restriction in the parietal white matter, splenium and genu of the corpus callosum without mass effect and slight thickening at the splenium of corpus callosum with no contrast enhancement. With empirical and support treatment, her neurological examination was completely normal by the 18th hour. The nasopharyngeal respiratory adenoviral PCR resulted positive. She was discharged with total clinical and radiological resolution on the 10th day of admission. The case was diagnosed with MERS type-2 which is rarely associated with adenoviral infection.
\end{abstract}

Conclusion. This report is the first case of adenovirus related MERS type- 2 in a Turkish child. Pediatricians, child neurologists, child infection specialists and radiologists should recognize this condition to ensure appropriate diagnosis.

Key words: encephalopathy, corpus callosum, adenovirus, child.

Mild encephalopathy with a reversible splenial lesion (MERS) is a clinic-radiological syndrome characterized by transient mild encephalopathy and various magnetic resonance imaging (MRI) findings including transient lesions in the splenium of the corpus callosum. ${ }^{1}$ MERS has two radiological subtypes based on imaging findings. In MERS type-1 reversible lesions are limited to the splenium of corpus callosum whereas, in type-2, lesions extend to the splenium of the corpus callosum and might involve the entire corpus callosum, subcortical

$\bowtie \quad$ Mustafa Emre Akın

dremreakin@gmail.com

Received 15th April 2020, revised 8th July 2020,

26th July 2020, accepted 7th September 2020. white matter near central sulcus. ${ }^{2}$ The syndrome is characterized by a prodromal period of 1-7 days, followed by encephalopathy which may be accompanied by behavioral change, seizures and hallucinations. The syndrome mainly affects children and young adults and the prognosis is generally good with complete or nearly complete clinical resolution over days without treatment. Radiological recovery can last days to weeks. ${ }^{3}$ The exact pathophysiology is unknown. Viral infections are found to be the most common associated etiological factor in childhood. ${ }^{4}$ To the best of our knowledge, there are only three published cases of MERS type2 associated with adenovirus infection. 5,6 We present here the first case of adenovirus related MERS type-2 in a Turkish child and a literature review on adenovirus related MERS. 


\section{Case Report}

A previously healthy 10-year-old girl was admitted to our hospital with slurred speech and confusion. She had had a fever for the last three days. She had not received any medication aside from acetaminophen and cefaclor for the last three days. She had no history of neurologic disorders or developmental delays. The family history was unremarkable and there was no consanguinity between parents. On admission, she had hyperemia in the oropharynx and neurological examination revealed lethargy in the following hours. There were no signs of focal neurologic deficit or meningeal irritation. Laboratory investigations revealed: hemoglobin $14.3 \mathrm{mg} / \mathrm{dl}$, white blood cell count $6510 / \mathrm{mm}^{3}$ and platelets $179000 / \mathrm{mm}^{3}$; C reactive protein (CRP) $64,10 \mathrm{mg} / \mathrm{L}(0-5 \mathrm{mg} / \mathrm{L})$, serum glucose level $98 \mathrm{mg} / \mathrm{dl}$, Na $136 \mathrm{mmol} / \mathrm{L}$, and K $3.4 \mathrm{mmol} / \mathrm{L}$. Serum biochemical investigations were within normal range. There were no leukocytes on the microscopic examination of the cerebrospinal fluid (CSF). The concentration of CSF protein and glucose levels were $16.4 \mathrm{mg} / \mathrm{dl}(15-45 \mathrm{mg} /$ dl) and $55.4 \mathrm{mg} / \mathrm{dl}$ respectively. On the day of admission, an emergency cranial computer tomography evaluation was unremarkable. The brain MRI revealed bilateral diffusion restriction in parietal white matter, splenium and genu of corpus callosum without mass effect and slight thickening at the splenium of the corpus callosum with no contrast enhancement (Fig. 1). The patient was hospitalized and given empirical oseltamivir and acyclovir treatment. The electroencephalography (EEG) was consistent with generalized intermittent slow wave activity. At the $18^{\text {th }}$ hour of admission, the neurological exam was normal. The CSF bacterial culture and CSF herpes simplex virus-1 (HSV-1) and HSV-2 viral polymerase chain reaction (PCR) were negative. Tandem MS, serum and urine amino acid and organic acids, lactate and pyruvate levels were normal. The nasopharyngeal respiratory viral PCR resulted positive for adenovirus and negative for respiratory syncytial virus-A (RSV-A), RSV-B, rhinovirus, parainfluenza-1, parainfluenza-2, parainfluenza-3, parainfluenza-4 viruses, coronavirus, influenza A, influenza B, human metapneumovirus, human bocavirus, enterovirus, and parechovirus. On the $10^{\text {th }}$ day of admission, the neurological examination, brain MRI examination (Fig. 2) and EEG were completely normal. The child was discharged with no sequela on the $10^{\text {th }}$ day of admission. The clinical and radiological course of this case was discussed by a team composed of radiologists, pediatrician, child neurologist, and child infectious disease specialist and revealed the diagnosis of MERS type-2. The family provided informed consent for this report.

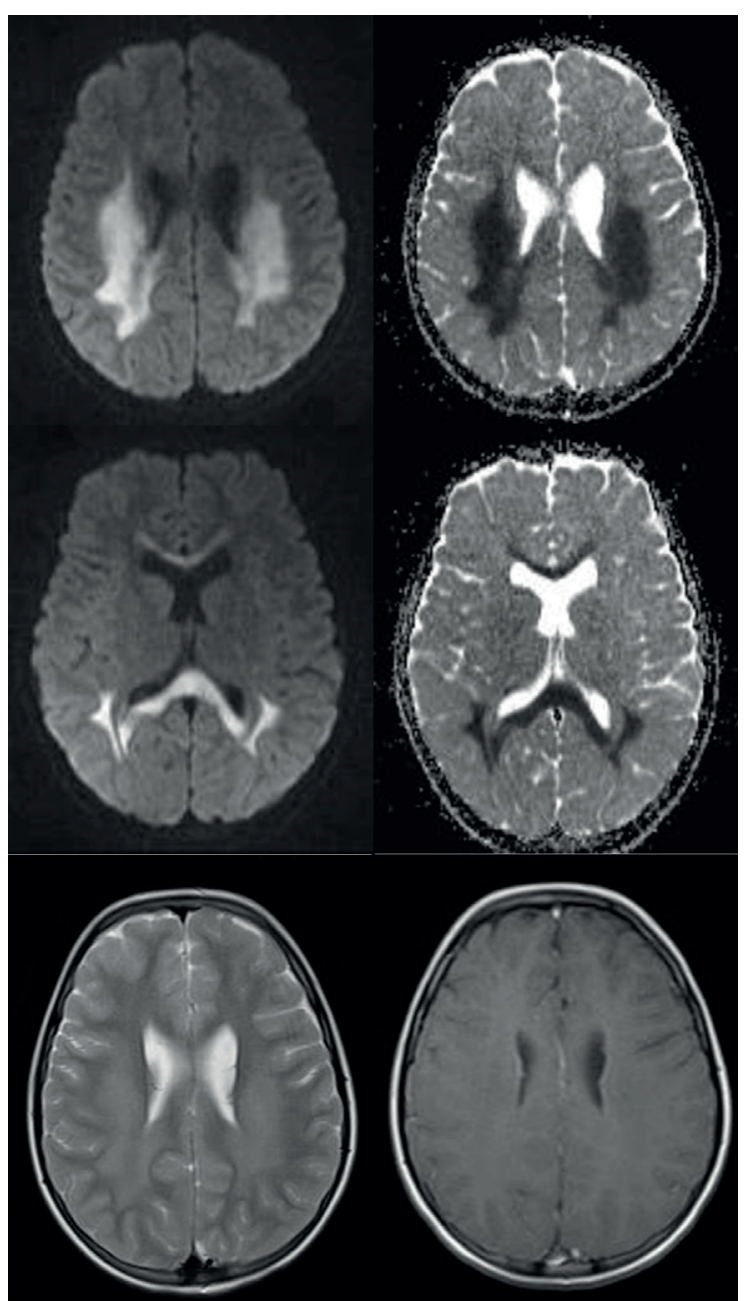

Fig. 1. Bilateral diffusion restriction in parietal white matter, splenium and genu of the corpus callosum without mass effect and slight thickening at the splenium of the corpus callosum at the admission. 


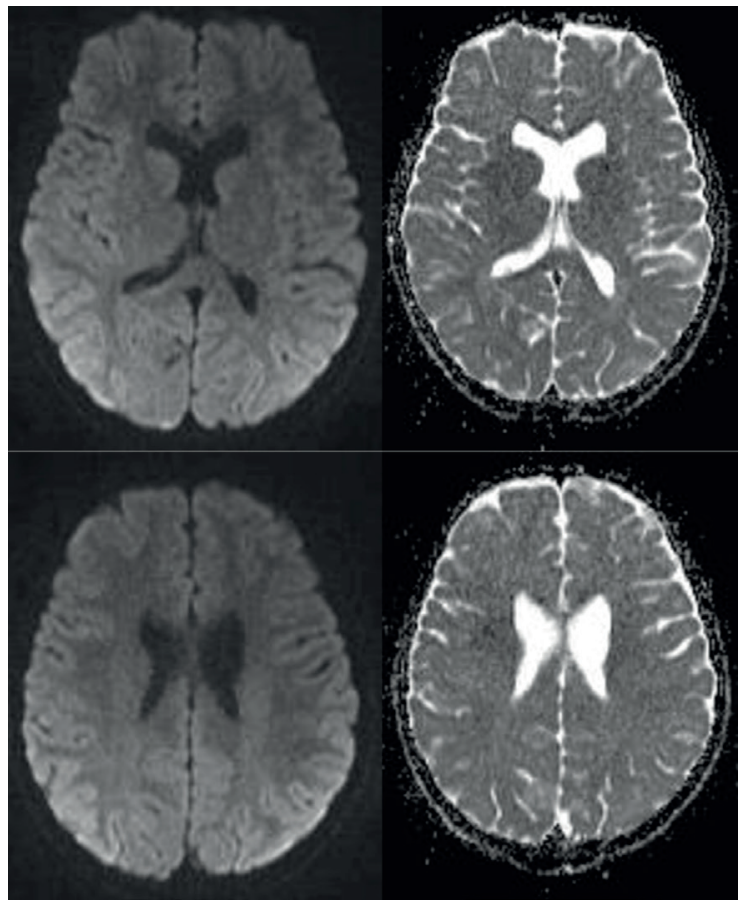

Fig. 2. Normal diffusion magnetic resonance imaging examination findings on the 10th day of admission.

\section{Discussion}

This report is the first case of adenovirus related MERS type-2 in a Turkish child who had extensive symmetrical white matter involvement on MRI. Clinical findings had resolved in a matter of hours and were followed by radiological findings on the $10^{\text {th }}$ day of admission.

In patients presenting with reversible splenial lesions, the diagnosis of MERS is established if the patient has an acute onset of impaired consciousness lasting for more than 12 hours, with no evidence of inflammatory changes in the cerebrospinal fluid. ${ }^{7}$ Acute disseminated encephalomyelitis (ADEM) which is a demyelinating disease of the central nervous system that typically presents as a monophasic disorder associated with multifocal neurologic symptoms and encephalopathy, should be considered in the differential diagnosis. ${ }^{8}$ Encephalopathy often develops rapidly and almost simultaneously with multifocal neurologic deficits after the onset of viral infections in matter of days to weeks while CSF analysis reveals mild pleocytosis. Although the involvement of the corpus callosum is frequent in ADEM, the distribution of the lesions is asymmetric contrary to the symmetric involvement seen in MERS. Resolution of white matter lesions may take weeks to months and require treatment with corticosteroids. The severe phase of ADEM typically lasts from two to four weeks. The patients' clinical condition may deteriorate and patients may also develop new neurologic signs during hospital admission. ${ }^{8}$ Diffuse axonal injury (DAI) is an another condition in which the corpus callosum involvement is frequent and should be considered in the differential diagnosis of MERS. DAI describes a process of widespread axonal damage that is observed after traumatic brain injury. ${ }^{9}$ The patients are usually comatosed at admission. DAI is pathologically defined by axonal damage in multiple regions of the white matter that often causes multiple neurological impairments. Brainstem, corpus callosum and subcortical white matter are the most common affected white matter regions. ${ }^{9}$ The patient in this report developed symptoms soon after the onset of adenovirus with no trauma history and also quickly recovered completely without corticosteroids. CSF analysis revealed normal cell count. Therefore these findings are unlikely to be manifestations of ADEM or DAI. Other differential diagnoses of the white matter lesions including splenium of the corpus callosum involve, posterior reversible encephalopathy syndrome, ischemia, multiple sclerosis and lymphoma are excluded clinically and radiologically. ${ }^{10}$

The exact pathophysiology of MERS is unknown. Proposed mechanisms of MERS include intramyelinic axonal edema, ${ }^{3}$ oxidative stress, ${ }^{12}$ immune system activation, ${ }^{11}$ elevated levels of IL-6 in which leads to antidiuretic hormone release, hyponatremia, systemic inflammation $^{13}$ and inflammatory infiltrates. ${ }^{1}$ The etiology of MERS is summarized in Table I. In our case, there was no hypoglycemia and hyponatremia. The metabolic tests excluded most of the inherited metabolic diseases. Our 
Table I. Etiology of mild encephalopathy with a reversible splenial lesion (MERS).

\begin{tabular}{ll}
\hline Etiology of MERS* & \\
\hline Infectious etiology & Non-infectious etiology \\
\hline Viral & Metabolic diseases \\
Influenza virus (most common) & Hypoglycemia \\
Rotavirus & Hyponatremia \\
Measles & Antiepileptics \\
Herpesvirus-6 & Antiepileptic drug withdrawal \\
Epstein-Barr virus & High attitude cerebral edema \\
Varicella zoster virus & Malnutrition \\
Mumps & Anorexia nervosa \\
Cytomegalovirus & Charcot-Marie-Tooth disease \\
Adenovirus & Marchiafava-Bignami disease \\
Bacterial & B12 deficiency \\
Salmonella enteritidis & Systemic lupus eritematozus \\
Legionella pneumophila & Side effect of chemotherapeutics \\
Escherichia coli & Methyl bromide exposure \\
& Diet pills \\
\hline$*$ Tada et al (2004) Garcia-Monco JC et al (2011) Karampatsas K et al (2015) Chen WX et al (2016) Feraco P et al (2018)
\end{tabular}

*Tada et al (2004), Garcia-Monco JC et al (2011), Karampatsas K et al (2015), Chen WX et al (2016), Feraco P et al (2018)

case had neither history of drug use other than antibiotics and acetaminophen nor a history of being at high altitude. Clinical and laboratory findings suggested that our patient has encephalopathy rather than encephalitis as the patient's CSF examination revealed no signs of inflammation. Hence the diagnosis of adenovirus associated MERS with extensive white matter involvement was established and supported by the resolution of clinical and radiological findings on the $10^{\text {th }}$ day of admission. Although our case and most cases reported in the literature with viral etiology depends on nasopharyngeal swabs, ${ }^{5,6,14}$ CSF adenoviral PCR would be very beneficial for our case to prove the etiology.

Human adenovirus is a ubiquitous, nonenveloped virus with linear double-stranded DNA and a known cause of febrile syndromes, mainly in early childhood. ${ }^{17}$ Adenovirus infection rarely involves the central nervous system in immunocompetent children. ${ }^{18}$

We performed a PubMed and a Google search with the following terms: "mild encephalopathy with reversible splenial lesion", "reversible splenial lesion", "adenovirus" "child" and "children". A total of 4 publications, from 2010 to 2016, were included in the review. Chen et al reported 15 MERS cases, one of the cases was a child with adenovirus related MERS type- $1 .{ }^{14}$ Fang et al. ${ }^{19}$ reported 29 children with MERS from China. This report included 2 children with adenovirus-related MERS. One of these children was diagnosed as MERS type-1 and positive for adenovirus, echovirus and coxachie virus. The second child was positive for only adenovirus and diagnosed as MERS type-2. ${ }^{19}$ Imamura et al. $^{5}$ reported 2- and 6-year-old sisters with MERS type-2 from Japan, in one of the patients, adenovirus was isolated from a nasopharyngeal swab. Although the other patient's viral etiology could not be determined, the authors diagnosed clinically both patients as adenovirus related MERS type-2. Ka et al. ${ }^{6}$ reported 7 children with MERS from Australia, of these 4 were MERS type-2 and one was associated with adenovirus. In total there have been only 3 reports identified which included adenovirus related MERS type2 cases in the literature. ${ }^{5,6,19}$

The mechanism of the radiological findings in MERS is not established clearly. MERS 
frequently involves splenium of the corpus callosum and seldomly extends to other corpus callosum parts and periventricular white matter which predicate subtype. ${ }^{7,8,20}$ Restricted diffusion in white matter and corpus callosum is thought to be the results of intramyelinic edema, interstitial edema in tightly packed fibers and transient inflammatory infiltrate. ${ }^{3,11}$ These lesions frequently involve only the splenium of the corpus callosum in reported cases and rarely periventricular white matter and other parts of the corpus callosum. In our case the MRI findings were consistent with type 2 MERS and the degree of white matter involvement was extensive even among the other reported MERS type-2 cases with various etiologies. ${ }^{6,7,14} \mathrm{Ka}$ et al. ${ }^{6}$ reported no correlation between the extension of MRI findings and clinical symptoms or prognosis. Similarly, in our case, there was extensive white matter involvement in the MRI whereas, the child only had speech difficulties and lethargy which resolved in 18 hours.

In conclusion, MERS should be considered in the differential diagnosis of children presenting with neurological findings and reversible splenial lesions. Child neurologists, pediatricians, radiologists and child infectious disease specialists should keep in mind that MERS type-2 can be associated with different infectious agents including adenovirus.

\section{Author contribution}

Authors confirm contribution to the paper as follows:

Mustafa Emre Akın analyzed and interpreted the patient data regarding the disease and drafted the manuscript. Ayşegül Neşe Çıtak Kurt performed the neurological examination, Gülsüm İclal Bayhan and Zeynep Dinçer Ezgü interpreted the infection markers, Mustafa Emre Akın and Sevtap Şimşek Bulut evaluated the MRI examination of the case, and all authors had major contributions in writing the manuscript. All authors read and approved the final manuscript.

\section{Conflict of interest}

The authors declare no conflict of interests.

\section{REFERENCES}

1. Tada H, Takanashi J, Barkovich AJ, et al. Clinically mild encephalitis/encephalopathy with a reversible splenial lesion. Neurology 2004; 63: 1854-1858.

2. Takanashi J, Imamura A, Hayakawa F, Terada $H$. Differences in the time course of splenial and white matter lesions in clinically mild encephalitis/ encephalopathy with a reversible splenial lesion (MERS). J Neurol Sci 2010; 292: 24-27.

3. Takanashi J. Two newly proposed infectious encephalitis/encephalopathy syndromes. Brain Dev 2009; 31: 521-528.

4. Garcia-Monco JC, Cortina IE, Ferreira E, et al. Reversible splenial lesion syndrome (RESLES): what's in a name? J Neuroimaging 2011; 21: e1-e14.

5. Imamura $\mathrm{T}$, Takanashi J, Yasugi J, Terada $\mathrm{H}$, Nishimura A. Sisters with clinically mild encephalopathy with a reversible splenial lesion (MERS)-like features; Familial MERS? J Neurol Sci 2010; 290: 153-156.

6. Ka A, Britton P, Troedson C, et al. Mild encephalopathy with reversible splenial lesion: an important differential of encephalitis. Eur J Paediatr Neurol 2015; 19: 377-382.

7. Kashiwagi M, Tanabe T, Shimakawa S, et al. Clinicoradiological spectrum of reversible splenial lesions in children. Brain Dev 2014; 36: 330-336.

8. Cole J, Evans E, Mwangi M, Mar S. Acute disseminated encephalomyelitis in children: an updated review based on current diagnostic criteria. Pediatr Neurol 2019; 100: 26-34.

9. Su E, Bell M. Diffuse axonal injury. In: Laskowitz D, Grant G (eds) Translational Research in Traumatic Brain Injury (1st ed) Boca Raton: CRC Press/Taylor and Francis Group, 2016.

10. Friese SA, Bitzer M, Freudenstein D, Voigt K, Küker W. Classification of acquired lesions of the corpus callosum with MRI. Neuroradiology 2000; 42: 795802.

11. Shi BC, Li J, Jiang JW, Li MX, Zhang J, Shang XL. Mild encephalitis/encephalopathy with a reversible splenial lesion secondary to encephalitis complicated by hyponatremia: a case report and literature review. Medicine (Baltimore). 2019; 98: e17982.

12. Miyata R, Tanuma N, Hayashi M, et al. Oxidative stress in patients with clinically mild encephalitis/ encephalopathy with a reversible splenial lesion (MERS). Brain Dev 2012; 34: 124-127. 
13. Swart RM, Hoorn EJ, Betjes MG, Zietse R. Hyponatremia and inflammation: the emerging role of interleukin-6 in osmoregulation. Nephron Physiol 2011; 118: 45-51.

14. Chen WX, Liu HS, Yang SD, et al. Reversible splenial lesion syndrome in children: retrospective study and summary of case series. Brain Dev 2016; 38: 915-927.

15. Karampatsas K, Spyridou C, Morrison IR, Tong CY, Prendergast AJ. Rotavirus-associated mild encephalopathy with a reversible splenial lesion (MERS)-case report and review of the literature. BMC Infect Dis 2015; 15: 446.

16. Feraco P, Porretti G, Marchiò G, Bellizzi M, Recla M. Mild encephalitis/encephalopathy with reversible splenial lesion (MERS) due to cytomegalovirus: case report and review of the literature. Neuropediatrics 2018; 49: 68-71.
17. Smith JG, Wiethoff CM, Stewart PL, Nemerow GR Adenovirus. Curr Top Microbiol Immunol 2010; 343: 195-224.

18. Tebruegge M, Curtis N. Adenovirus: an overview for pediatric infectious diseases specialists. Pediatr Infect Dis J 2012; 31: 626-627.

19. Fang Q, Chen L, Chen Q, Lin Z, Yang F. Clinically mild encephalitis/encephalopathy with a reversible splenial lesion of corpus callosum in Chinese children. Brain Dev 2017; 39: 321-326.

20. Yıldız AE, Maraş Genç H, Gürkaş E, Akmaz Ünlü H, Öncel İH, Güven A. Mild encephalitis/ encephalopathy with a reversible splenial lesion in children. Diagn Interv Radiol 2018; 24: 108-112. 